\title{
Problématique de la valorisation agricole des biodéchets dans la ville de Lubumbashi : identification des acteurs, pratiques et caractérisation des déchets utilisés en maraîchage
}

\author{
Useni Sikuzani Yannick1, Baboy Longanza Louis ${ }^{182}$, Kanyenga Lubobo Antoine ${ }^{1}$, Assani Bin \\ Lukangila1, Mbuyi Kabengele Matthieu ${ }^{1 *}$, Kasanda Mukendi Nathan ${ }^{1}$, Mbayo Kyongo Longo Jerry3, \\ Mpundu Mubemba Michel ${ }^{1}$, Nyembo Kimuni Luciens ${ }^{1}$ \\ ${ }^{1}$ Faculté des Sciences Agronomiques, Université de Lubumbashi, BP 1825 Lubumbashi, RD Congo ; \\ 2 École interfacultaire de bioingénieurs, Faculté des Sciences, Université Libre de Bruxelles, Belgique ; \\ ${ }^{3}$ Ecole supérieure des Ingénieurs Industriels, Université de Lubumbashi, BP 1825 Lubumbashi, RD Congo \\ Auteur correspondant : yannickuseni@gmail.com, +243813666582
}

Original submitted in on $13^{\text {th }}$ January 2014. Published online at www.m.elewa.org on $30^{\text {th }}$ April 2014. http://dx.doi.org/10.4314/jab.v76i1.5

\section{RESUME}

Objectifs : une enquête a été initiée en vue de décrire le profil des maraichers, la variabilité des pratiques liée à cette activité et l'analyse des valeurs fertilisantes relatives à chacun des types des biodechets utilisée en agriculture urbaine.

Méthodologie et résultats: La détermination du profil des acteurs était soumise à une analyse en composante principale (ACP) alors que les paramètres réunis autour de la pratique de maraîchage ont été analysés par le test khi deux. Par ailleurs, la composition physico-chimique des différents types de biodechets a été analysée pour en ressortir la valeur fertilisante relative. Les résultats obtenus montrent que les maraichers de Lubumbashi sont essentiellement des femmes mariées, âgés de plus de 18 ans, actrices de carrière et ne possédant pas de titre foncier. La moitié des biodéchets utilisés était des fumiers de poule et accessoirement le fumier de porc, obtenus par achat. Les maraichers préfèrent une application à l'état brut, qu'ils enfouissent dans le sol. En épandant les biodéchets au sol, $33 \%$ des maraichers s'attendent à une augmentation de productivité et une amélioration des propriétés physico-chimiques du sol contre $48,3 \%$ à l'amélioration de l'aspect végétatif des légumes et l'augmentation de rendement. La composition des types de biodéchets a varié en fonction des éléments considérés, tout comme le rapport $\mathrm{C} / \mathrm{N}$ qui leurs est associé.

Conclusion et application de la recherche : La gestion des déchets urbains constituant un problème majeur dans les pays en développement, l'identification des contraintes à leur valorisation en agriculture urbaine présente une perspective intéressante et une réponse aux agriculteurs.

Mots-clés : Agriculture urbaine, valorisation, biodéchets, maraichers, Lubumbashi 


\begin{abstract}
Issue of agricultural use of bio-waste in the city of Lubumbashi: identification of stakeholders, practices and waste characterization.

Objectives: An investigation was initiated to describe the profile of the stakeholders, the variability of the practices and analysis of the fertilizing values for each type of biowaste used in urban agriculture. Methodology and results: Determining the profile of the stakeholders was subjected to principal component analysis (PCA), while the parameters gathered around the practice of gardening were analyzed by chisquare test. Moreover, the physico- chemical composition of different types of bio-waste was analyzed to highlight the fertilizer relative value. The results obtained show that the market gardeners of Lubumbashi are mainly married women, aged over 18 , and having no land title. Half of biowaste used was chicken manure and pig manure, obtained by purchase and come from the farms. The market gardeners prefer an application in the rough, they burrow into the soil. By spreading biowaste in soil, 33\% market gardeners expect increased productivity and improved physico- chemical properties of soil against $48.3 \%$ to improve the vegetative aspect of vegetables and increasing yield. The composition of the types of biowaste has varied depending on the elements considered, like that their associated $\mathrm{C} / \mathrm{N}$ ratio.

Conclusion and application: The urban waste management constitutes a major problem in developing countries, identifying constraints to their development in urban agriculture presents an interesting perspective and a response to farmers.
\end{abstract}

Keywords: Urban Agriculture, valorization, biowaste, market gardeners, Lubumbashi

\section{INTRODUCTION}

L'urbanisation et le développement économique ont généralement pour corollaire une augmentation de la production des déchets par habitant, et un accroissement des besoins alimentaires ; II devient donc impératif de mettre en place, surtout dans les villes africaines, un système de gestion des déchets solides qui pourrait favoriser le développement de l'agriculture urbaine (N'Dienor, 2006). Depuis une dizaine d'années, l'agriculture urbaine est reconnue comme un enjeu majeur en termes d'approvisionnement des villes, d'emploi et de gestion de l'environnement urbain (Houot et al., 2003). Confronté à la demande urbaine en augmentation, au manque d'infrastructures de transport et à la faible productivité de l'agriculture dans les zones rurales, l'agriculture dans la ville de Lubumbashi s'étend de plus en plus sur les ferralsols, présentant une faible potentialité de production primaire au regard de leurs propriétés chimiques (Kasongo et al., 2013). D'où, dans plusieurs villes d'Afrique les biodéchets sont utilisés pour élever la productivité des sols et les rendements des cultures. Cependant, s'il existe différents travaux sur les effets des biodéchets sur les propriétés des sols et sur les rendements de différentes cultures (Ndienor, 2006 ; Mulaji, 2011 ; Useni et al, 2012; Useni et al., 2013), peu de travaux présentent les différents acteurs de l'agriculture péri-urbaine et leurs perceptions de la fertilité de leurs sols et de leurs attentes en ce qui concerne les biodéchets (Kaboré et al., 2011). Un accompagnement des acteurs de l'agriculture urbaine nécessite une meilleure connaissance des contraintes et de leurs attentes. Néanmoins, dans la ville de Lubumbashi, les outils de diagnostic des problèmes de cette agriculture et les moyens d'intervention pour son développement durable font défaut aux chercheurs, décideurs et agents du développement (SENAHUP, 2008). II est devenu évident qu'il faut s'assurer que les agriculteurs comprennent très bien les interdépendances entre les caractéristiques et la fertilité du sol, et leurs connaissances, perceptions et attitudes sont considérées de plus en plus comme une ressource importante dans la compréhension mutuelle et le développement participatif des technologies (Kaboré et al., 2011).Le présent travail a pour 
objectif général de déterminer la Problématique de la valorisation agricole de déchets urbains solides dans la ville de Lubumbashi (cas de maraichers). Les objectifs spécifiques s'articule autour de (i) l'obtention des informations sur les différents acteurs de l'agriculture urbaine et périurbaine

\section{MATÉRIEL ET MÉTHODES}

Milieu d'étude : Appelée capitale du cuivre, la ville de Lubumbashi est le chef lieu de la province du Katanga localisée à $11^{\circ} 39^{\prime}$ sud et $27^{\circ} 28^{\prime}$ est de la République démocratique du Congo. Elle est actuellement composée de 42 quartiers répartis sur 7 communes (maraichers) par une enquête; (ii) l'échantillonnage et la caractérisation des biodéchets utilisés par les acteurs; et (iii) la confrontation les critères analytiques sur les biodéchets avec les attentes des utilisateurs.

(Nkuku et Remon, 2006): Lubumbashi, Kenya, Kampemba, Katuba, Kamalondo, Ruashi et Annexe, commune regroupant l'habitat en périphérie de la ville (couronne autour d'autres communes).

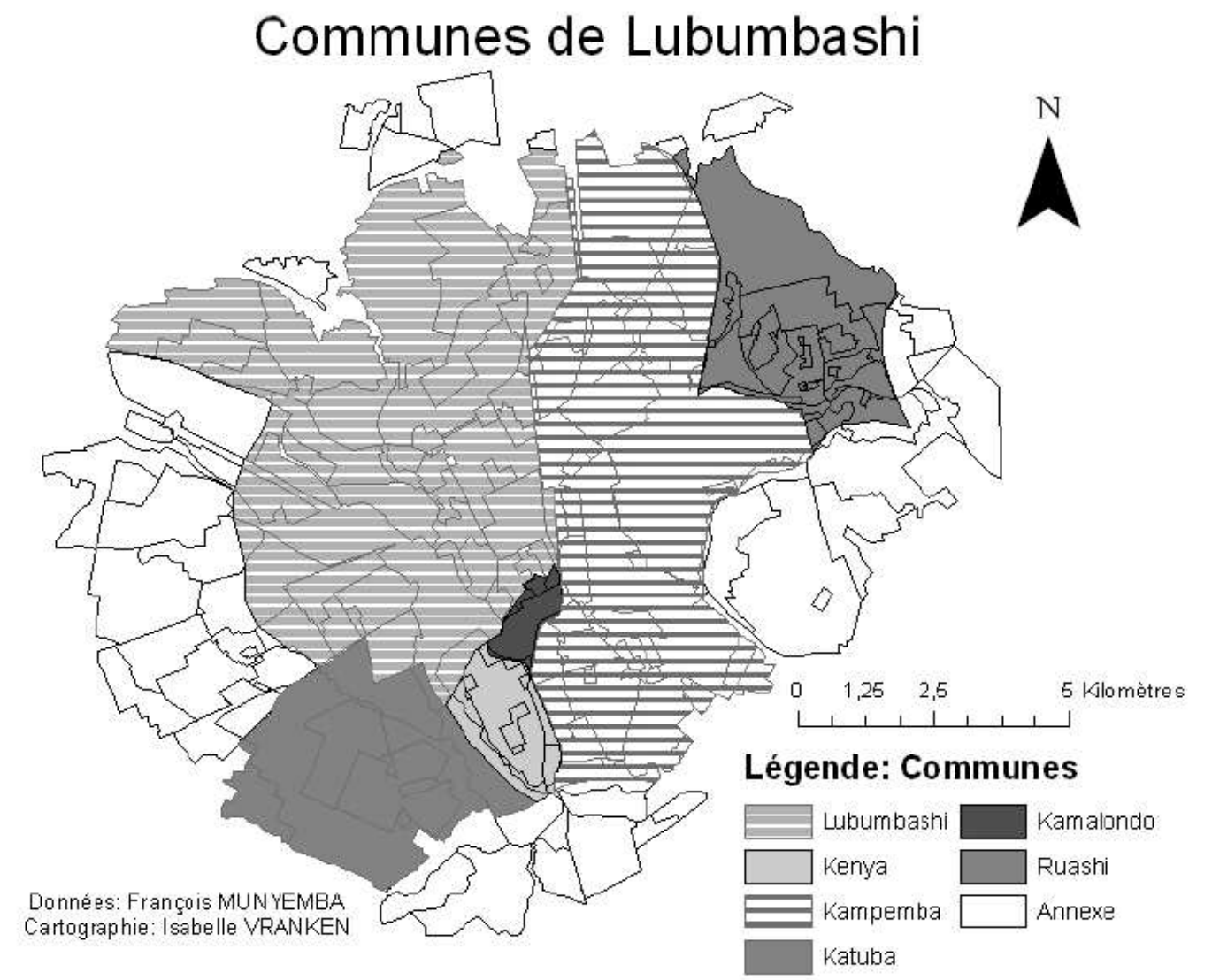

Figure 1 : Subdivision administrative de la ville de Lubumbashi (Vranken, 2010)

Le climat de Lubumbashi est du type CW6, selon le système de classification de Koppen, caractérisé par une saison des pluies (de novembre à mars), une saison sèche (mai à septembre) et deux mois de transition (Avril à Octobre). Les précipitations annuelles s'élèvent à $1270 \mathrm{~mm}$ avec les valeurs extrêmes de 717 et $1770 \mathrm{~mm}$. La température moyenne annuelle est d'environ $20^{\circ} \mathrm{C}$ (Malaisse, 1990). La végétation primaire de la ville de Lubumbashi est la forêt claire du type Miombo. Cependant, à cause des activités anthropiques, cette forêt claire, est dans la région périurbaine, remplacée par une végétation secondaire, constituée par une savane (Mujinya et al., 2011). De nombreux habitants urbains étaient issus du Kasaï, de 
l'Angola et de la Rhodésie du nord. Les Lushois cultivaient surtout le manioc, le maïs-grain, le maïs vert, la patate douce, le sorgho, l'éleusine, l'arachide et diverses plantes légumières. Deux mille cinq cent cinquante travailleurs étaient employés dans les fermes (Sys et Schmitz, 1959). Avec une population d'environ 1500000 habitants en 2009 (INS, 2009) contre 1200000 en 2006 (Nkuku \& Remon, 2006), Lubumbashi est la deuxième ville la plus peuplée après Kinshasa. Les principales sources de revenus des ménages Lushois sont le salaire, les petits commerces et la propriété. La plupart des Lushois qui reçoivent un salaire ne le touchent qu'irrégulièrement. L'agriculture avait subit de plein fouet les effets de la politique de la zairianisation en 1973 et ne s'est jamais remise de la mauvaise gestion qui s'ensuivit (Frauman, 2003). Depuis la chute de la Gécamines, l'essentiel de l'agriculture n'est resté qu'une activité de subsistance. Principalement vivrière, sa production insuffisante ne peut pas nourrir l'ensemble de la population (Kalamba et al., 1998).

Matériels : Pour une bonne évolution de la recherche, une fiche d'enquête a été élaborée; cette dernière est composée d'une série de 22 questions. Les paramètres relevés ont concerné : l'identification de l'acteur et de son exploitation (âge, niveau d'instruction, superficies exploitées, accessibilité au foncier), la perception et la gestion de la fertilité par l'acteur (connaissance, évaluation et gestion de la fertilité sur la parcelle), les attentes des acteurs en termes de qualité des substrats organiques et leurs critères d'évaluation de cette qualité. Les déchets urbains solides ont été obtenus sur les différents sites maraichers de la ville de Lubumbashi (tableau 1)

Tableau 1. Liste des différents déchets urbains solides prélevés chez les maraichers et sites de prélèvement

\begin{tabular}{ll}
\hline Déchets urbains solides & Sites de prélèvement \\
\hline Composte & Cité des jeunes et Magone \\
Fumier de porcs & Cité des jeunes, Magone et foyer Katuba \\
Fumier de poule & Cité des jeunes, Magone, Vinamon, Kassapa, Kabecha, \\
& Wamba, Athennée, Kalebuka et Kilobelobe \\
Paille & $\begin{array}{l}\text { Vinamon, Kabecha, Kalebuka, Athennée, Wamba, } \\
\text { Kassapa, Kilobelobe, Cité des jeunes et Magone } \\
\text { Kutres }\end{array}$ \\
& Kassapa, Kilobelobe, Athennée, Cité des jeunes et \\
& Magone \\
\hline
\end{tabular}

Méthodes: L'objectif de cette démarche est non seulement de comprendre la perception que les acteurs ont sur les déchets urbains solides, mais aussi de se rendre compte de leur pratique. Les enquêtes se sont déroulées en trois étapes :

- une tournée exploratoire dans la ville de Lubumbashi a permis de circonscrire davantage la zone d'étude ; -une phase d'enquête formelle à l'aide d'une fiche d'enquête (reprise en annexe) au cours de laquelle 32 paysans ont été enquêtés. Ces enquêtes ont été menées chez des exploitants dont les champs se situent sur dix sites et qui sont répartis de la façon suivante :

\begin{tabular}{|c|c|}
\hline * & site 1 (Magone) :1 enquêté \\
\hline 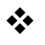 & site 2 (Cité des jeunes) : 1 enquêté ; \\
\hline$\%$ & site 3 (Kabecha) : 13 enquêtés ; \\
\hline$\star$ & site 4 (Wamba) :2 enquêtés ; \\
\hline$\%$ & site 5 (Vinamont) : 2 enquêtés : \\
\hline$*$ & site 6 (Kilobelobe) : 4 enquêtés ; \\
\hline$\star$ & site 7 (Kalebuka) : 1 enquêté ; \\
\hline
\end{tabular}

* $\quad$ site 8 (Athenné de la Katuba) : 5 enquêtés ;

* $\quad$ site 9 (foyer Katuba) : 2 enquêtés

* $\quad$ site10 (Kassapa) : 1 enquêté.

- Une dernière phase au cours de laquelle nous avons échantillonné les biodéchets.

L'objectif de la démarche est d'avoir un aperçu sur ce que les acteurs apportent dans leurs champs. Pour cela, nous avons identifié des tas de biodéchets déposés dans le champ pour l'amendement et avons procédé à un échantillonnage au moins $5 \mathrm{Kg}$. L'ensemble est mis dans des sacs et transporté dans la serre, où il est séché à l'air libre pendant 10jours. La caractérisation physique a consisté, après séchage des échantillons de biodéchets, au triage et séparation de différents constituants de ce dernier. Les différents constituants physiques groupés en cinq catégories (matière non biodégradable, fumier de poule, fumier de porc, composte, et la paille) ont été pesées. La proportion de chaque constituant dans le poids total de biodéchets a été trouvée par la formule suivante: 
Proportion du constituant= (poids du constituant/poids total de biodéchets) $\times 100$. La teneur en matière organique et en éléments minéraux ( $\mathrm{N}$ total, $\mathrm{P}$ total, $\mathrm{Ca}$, $\mathrm{Mg}$,) ont été déterminées suivant les méthodes d'analyse décrite par Mulaji (2011). Les résultats de l'enquête ont été traités à l'aide d'une analyse en composantes principales (ACP) pour voir quelle sont

\section{RÉSULTATS}

Profil des maraichers: La figure 2 indique trois groupes des maraichers sur un total des 10 sites enquêtés en fonction de profil à l'issue de l'analyse en composante principale (ACP). Le premier réunit les sites I, II, IV, V, VI et VII caractérisés par les acteurs de carrière, âgés de 25 à 35 ans, n'appartenant pas au les variables qui déterminent chaque site. En suite, un test de Chi-carré était réalisé sur les variables suivantes : le sexe, l'état civile, l'appartenance ou non à un groupe professionnel, l'activité secondaire, le statut foncier et la tranche d'âge des acteurs pour voir si la répartition de personnes dans différents groupes en fonction de leurs réponses était aléatoire ou non.

groupe professionnel et exerçant les activités connexes. Le second rassemble les maraichers du site III, âgés de 35 à 50 ans, du genre féminin, mariées, propriétaire de concessions. Enfin, le troisième groupe est constitué des maraichers du site VIII, n'ayant pas de statut foncier, masculins et célibataires.

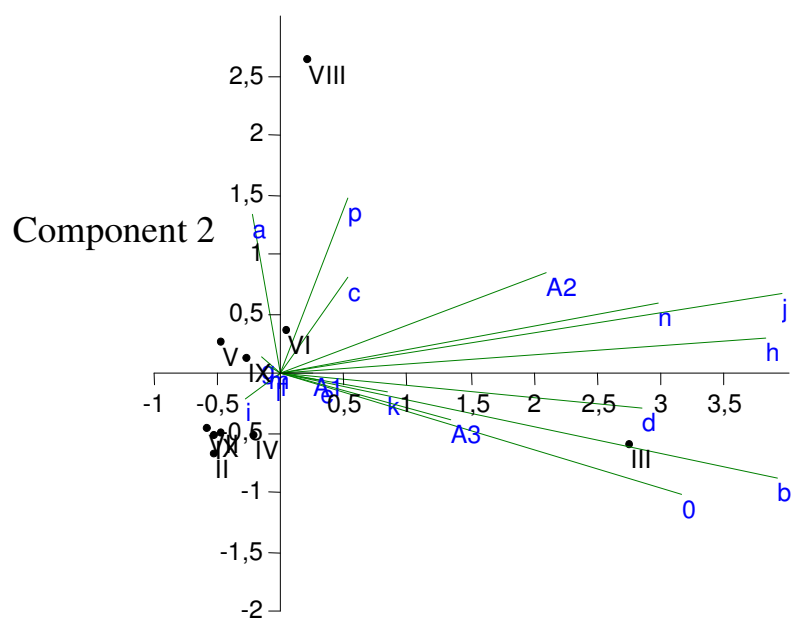

Component 1

Figure 2: Résultat analytique de l'ACP. Légende: sexe: masculin (a), féminin (b). État civile : célibataire(c), marié(d). Acteur de carrière (h), appartenance au groupe professionnel(i), non appartenance au groupe professionnel (j), aménagiste (k), activité connexes (n). Statu foncier : propriétaire (0), prêt (p). Tranche d'âge : 1825ans(A1), 25-35ans(A2).

Les résultats sur le profil des acteurs sont présentés dans le tableau 2. On remarque que les maraichers de sexe masculins se répartissent entre les groupes I $(71,8 \%)$ et III (100\%). Par contre, les maraichers de sexe féminins ne se rencontrent qu'aux deux premiers groupe, respectivement $28,2 \%$ (Groupe I) et $100 \%$ (groupe II). Quant à l'état civil, $59,3 \% ; 76,9 \%$ et $40 \%$ des maraichers enquêtés sont mariés; contre $40,7 \%$; $15,3 \%$ et $60 \%$ de célibataires, respectivement pour les trois groupes. L'agriculture urbaine à Lubumbashi est pratiquée par $40,6 \%$ et $20 \%$ d'acteurs de formation dans le premier et troisième groupe ; alors que 59,3 ; 100 et $80 \%$ de maraichers sont des acteurs de carrière. Du point de vue organisation professionnelle; on note que $43,7 \%$ des acteurs du premier et $100 \%$ des deux dernier groupes ne travaillent pas en équipe. Cependant, le seul groupe dont les acteurs (56,2\%) appartiennent à une organisation professionnelle est le Groupe I. 
Tableau 2. Profil des acteurs.

\begin{tabular}{|c|c|c|c|c|}
\hline Variables & & Gpl & Gpll & Gplll \\
\hline \multirow{2}{*}{ Sexe } & Masculin & 71,8 & 0 & 100 \\
\hline & Féminin & 28,1 & 100 & 0 \\
\hline \multirow{4}{*}{ État civil } & Célibataire & 40,6 & 15,3 & 60 \\
\hline & Marié(e) & 59,3 & 76,9 & 40 \\
\hline & Veuve & 0 & 7,6 & 0 \\
\hline & Veuf & 0 & 0 & 0 \\
\hline \multirow{2}{*}{ Formation } & $\mathrm{AF}$ & 40,6 & 0 & 20 \\
\hline & $\overline{A C}$ & 59,3 & 100 & 80 \\
\hline \multirow{2}{*}{ Organisation professionnelle } & AGP & 56,2 & 0 & 0 \\
\hline & NAGP & 43,7 & 100 & 100 \\
\hline \multirow{4}{*}{ Activités secondaires } & $\mathrm{Am}$ & 25 & 23 & 0 \\
\hline & Com & 18,7 & 0 & 0 \\
\hline & Étudiant & 12,5 & 0 & 0 \\
\hline & A Con & 43,7 & 76,9 & 100 \\
\hline \multirow{2}{*}{ Statut foncier } & Pro & 53,1 & 84,6 & 0 \\
\hline & LoC & 46,8 & 15,3 & 100 \\
\hline \multirow{2}{*}{ Tranche d'âge } & $18-25$ ans & 9,3 & 7,6 & 0 \\
\hline & $25-35$ ans & 90,7 & 92,4 & 100 \\
\hline
\end{tabular}

Légende : Acteur de formation (AF), acteur de carrière (AC), appartenance au groupe professionnel (AGP), non appartenance au groupe professionnel (NAGP), aménagiste (Am), commerçant (Com), activité connexe (A Con), propriétaire (Pro), locataire (Loc). Tranche d'âge : 18-25ans (A1), 25-35ans (A2).

Par rapport aux activités secondaires, le premier groupe des maraichers est le plus prépondérant en termes des types d'activités connexes (aménagistes, commerçants, conducteurs et étudiants). Par ailleurs, les maraichers possèdent un statut foncier, excepté ceux du troisième groupe, qui ne sont pas propriétaires de terrains mis en exploitation. On dénombre $53,1 \%$ et $84 \%$ des maraichers à titre foncier, respectivement pour les groupes I et II. La répartition d'âges des maraichers traduit exclusivement des maraichers dont la tranche d'âges se situant entre 25-35 ans pour le troisième groupe. A l'opposé les tranches d'âge de 1825 ans et 25-35 ans se repartissent, respectivement entre le groupe I $(9,3 \%$ et $90,7 \%)$ et le groupe II $(7,6 \%$ et $92,4 \%)$.

Mode d'obtention, source et gestion des biodéchets par les maraichers: Les paramètres relatifs à l'obtention, la source de provenance et la gestion des déchets urbains solides ont varié d'un maraicher à l'autre (Tableau 3). II ressort de l'analyse statistique (Khi deux $=51,767 ; \mathrm{DL}=34, \mathrm{p}=0,0453$ ), que ces paramètres ont pas varié en fonction des groupes des maraichers. Autrement dit, la répartition de variables expliquant le mode d'obtention, source et gestion de biodéchets par les acteurs sur les trois groupes n'est pas aléatoire. D'une manière générale, les biodéchets sont obtenus par achat, la majorité provenant de la ferme. Les maraichers pratiquent l'application des biodéchets an culture au choix, c'està-dire que l'utilisation est relative pour la plupart. Une exception est ressentie au niveau du groupe III, qui applique les biodéchets obligatoirement en maraichage. Par rapport au traitement des déchets, la grande partie des déchets ne passe pas par le compostage, sauf au premier où les biodéchets utilisés sont préalablement compostés. Sous les mêmes conditions, les déchets sont généralement enfouis dans le sol, sauf au deuxième groupe qui pratique alternativement l'enfouissement et l'incinération. En rapport avec la gestion des biodéchets après récolte, les deux premiers groupes pratiquent les recyclages des déchets, alors que le troisième groupe les incinère absolument. 
Tableau 3 : Mode d'obtention, source et gestion des biodéchets par les maraîchers

\begin{tabular}{|l|l|l|l|l|l|l|l|l|l|l|l|l|l|l|l|l|l|l|l|l|l|}
\hline GP & \multicolumn{3}{|c|}{ M. Obt } & \multicolumn{3}{l|l|}{ Source de prov } & \multicolumn{3}{l|}{ Fréq. ut } & \multicolumn{3}{l|}{ Compostage } & \multicolumn{3}{l|}{ Mode d'ut } & \multicolumn{3}{|c|}{ Gestion des résibiodéchets } \\
\hline & A & B & C & D & E & F & G & H & I & J & K & L & M & N & O & P & Q \\
\hline I & 69 & 32 & 0 & 66 & 3,1 & 32 & 44 & 56 & 53 & 47 & 75 & 6,2 & 19 & 41 & 3,1 & 31 & 25 \\
\hline II & 69 & 31 & 15 & 69 & 15 & 0 & 0 & 100 & 38 & 62 & 38 & 38 & 23 & 7,6 & 15 & 69 & 7,6 \\
\hline III & 60 & 40 & 0 & 80 & 0 & 20 & 60 & 40 & 20 & 80 & 80 & 20 & 0 & 0 & 0 & 0 & 100 \\
\hline
\end{tabular}

Légende : mode d'obtention: Achat(A), gratuite(B). Source de provenance : Curage(C) ; ferme(D); recyclage et curage $(E)$; menuiserie, ferme, curage et recyclage $(F)$; fréquence d'utilisation : Condition sine qua non(G), Condition non sine qua non $(\mathrm{H})$. Compostage $(\mathrm{I})$. Non compostage $(\mathrm{J})$. Mode d'utilisation : Enfouir $(\mathrm{K})$; incinérer $(\mathrm{L})$; épandage à la surface $(\mathrm{M})$. Gestion de résibiodéchets de récolte: Alimentation de bétail( $(\mathrm{N})$; vente $(0)$; recyclage sur la parcelle(P) et source d'énergie $(Q)$.

Quantité de biodéchets utilisée par are: D'une manière générale, les maraichers appliquent en culture le fumier de poule et accessoirement la paille. Ce constat se vérifie aux deux derniers groupes, alors que le premier groupe préfère le compost, mais aussi dans une moindre mesure, le fumier de porc (Figure 3).

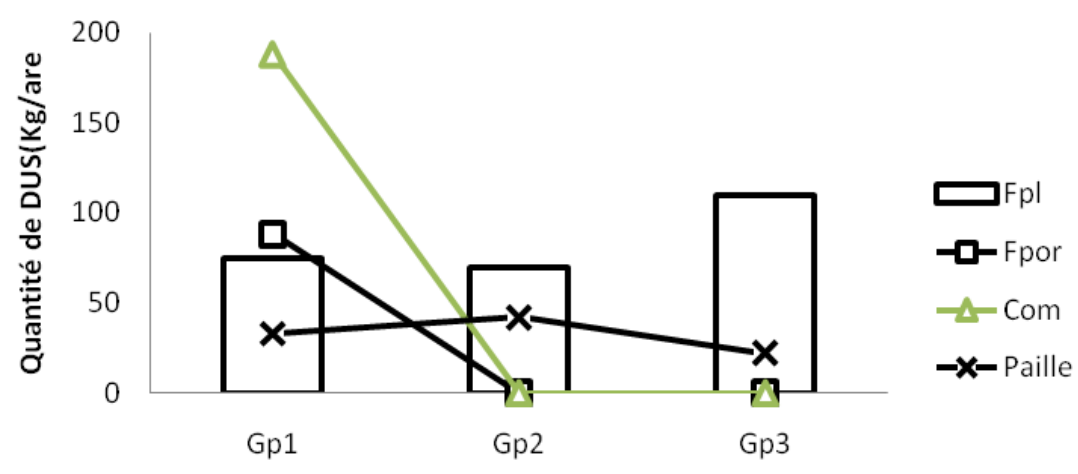

Groupes

Figure 3. Variation de quantités de biodéchets appliquée par are en fonction des types et des groupes des maraichers.

Attentes des différents acteurs et utilisations des composts déchets urbains: En utilisant les biodéchets, les maraichers s'attendent à l'augmentation de productivité et l'amélioration des propriétés physicochimiques du sol; tandis que le premier groupe se focalise principalement sur l'augmentation de la production des légumes. Le premier et le denier groupes évaluent la fertilité des biodéchets à travers l'aspect végétatif des légumes, alors que le second groupe associe à l'aspect végétatif à la productivité (tableau 4).

Tableau 4 : Variation des attentes et appréciation des biodéchets (\%) selon les groupes

\begin{tabular}{c|ccccc|ccc}
\hline Groupes & \multicolumn{5}{c|}{ Attentes } & \multicolumn{3}{c}{ Appréciation } \\
\hline \multirow{3}{*}{ I } & AP & AP \& APP & AP \& APC & APPC & AP \& APPC & AV & P & AV \& P \\
\cline { 2 - 10 } II & 36 & 14 & 14 & 14 & 21 & 43 & 21 & 36 \\
III & 23 & 0 & 15 & 15 & 38 & 23 & 7,6 & 69 \\
\hline
\end{tabular}


Légende : Augmentation de production(AP) ; Augmentation de production et amélioration de propriétés physique du sol (AP \& APP) ; Augmentation de production et amélioration de propriétés chimiques du sol (AP \& APC) ; amélioration de propriétés physiques et chimiques du sol (APPC); Augmentation de production, amélioration de propriétés physiques et chimiques du sol (AP \& APPC). Appréciation de la qualité : Aspect végétatif (AV); productivité $(\mathrm{P})$, Aspect végétatif et productivité (AV \& P).

Les caractéristiques physico-chimiques des biodéchets : Les teneurs en carbone organique total (COT) des substrats organiques prélevés chez les différents maraichers enquêtés ont montré une grande variabilité (Tableau 5). De tous les types de biodéchets considérés, le fumier de poules est le plus enrichi en carbone $(19,4 \%)$ et présente un $\mathrm{C} / \mathrm{N}$ plus élevé $(44,6 \%)$. En rapport avec les teneurs en $\mathrm{Ca}$, en matière minérale et en phosphore, le compost est plus riche que les autres types $(12,6 \% ; 80 \%$ et $0,38 \%)$. Le fumier de porc présente une teneur supérieur en $\mathrm{Mg}(22,9 \%)$ face aux restes des types des biodéchets considérés. La teneur en azote total est considérable dans la paille $(3,7 \%)$. Le rapport $\mathrm{C} / \mathrm{N}$ le plus faible est enregistré avec le fumier de porc $(5,3 \%)$, le plus élevé avec le fumier de poule $(44,6 \%)$; alors que le plus efficient serait celui de compost (12\%).

Tableau 5 : Composition des biodéchets selon les types. F=fumiers

\begin{tabular}{|l|r|r|r|r|}
\hline Constituants & Paille & Compost & F.Poules & F.Porcs \\
\hline $\mathrm{Ca}(\%)$ & 11,197 & 12,614 & 3,564 & 5,007 \\
\hline $\mathrm{Mg}(\%)$ & 16,34 & 20,81 & 9,857 & 22,909 \\
\hline $\mathrm{M} . \mathrm{O}(\%)$ & $\mathrm{RAS}$ & 19,178 & 33,402 & 7,428 \\
\hline $\mathrm{N}(\%)$ & 3,762 & 0,922 & 0,435 & 0,806 \\
\hline $\mathrm{P}(\%)$ & 0,206 & 0,388 & 0,331 & 0,283 \\
\hline $\mathrm{H}^{\circ}(\%)$ & 10 & 10 & 10 & 10 \\
\hline $\mathrm{M} \mathrm{minérales}(\%)$ & 68,608 & 83,032 & 19,419 & 69,406 \\
\hline $\mathrm{C}(\%)$ & $\mathrm{RAS}$ & 11,15 & 19,419 & 4,318 \\
\hline $\mathrm{C} / \mathrm{N}$ & - & 12,093 & 44,643 & 5,358 \\
\hline
\end{tabular}

\section{DISCUSSION}

Les axes 1 et 2 révèlent qu'il y a plus des femmes mariées qui pratiquent le maraîchage. En effet, recourant aux cultures de légumes de façon à compléter les revenus du ménage. Elles ne comptent que sur leurs économies personnelles, pour maintenir leur activité et le recouvrement partiel de leurs dépenses quotidiennes. Cela souligne également une situation plus fébrile, d'autant plus qu'elles n'exercent pas d'autre activités (Carole, 2012). Lekane (2003) et Niang (1999) ont également noté une grande proportion des femmes comme actrice dans le maraichage urbain à Yaoundé. L'Agriculture urbaine à Lubumbashi est menée en général par des jeunes. Néanmoins, il est clair que la pyramide d'âge des acteurs se situe entre 18-35 ans. Niang (1999) appuie ce constat en stipulant l'importance de la jeunesse des différents sites sur l'Agriculture urbaine, laquelle activité constitue une source d'emploi non négligeable au Sénégal pour les populations urbaines. D'après l'analyse d'ordination (Figure 1.), la majeure partie des maraichers sont acteurs de carrière. Ainsi une part importante d'acteurs présente un niveau scolaire du secondaire mais dans une option autre que l'agriculture (Carole, 2012). En outre, il paraît évident que, le pourcentage de maraîchers n'ayant reçu aucune formation est faible; alors qu'en général ils sont peu organisés du point de vue professionnel (Kabore, 2004). L'insécurité foncière dans laquelle se trouvent les acteurs lushois semble donc être moins forte. En termes de localisation, De Bon et al. (2008) avaient mis en évidence qu'au sein des villes Burkinabés, les maraîchers démarraient leur activité sur de petits espaces, dans les moindres interstices afin de faire face à cette insécurité foncière. Cette observation est confirmée dans le cas des maraichers enquêtés; dont la superficie moyenne des parcelles ne dépasse pas les $1000 \mathrm{~m}^{2}$ (respectivement $324,9 \mathrm{~m}^{2}, 475 \mathrm{~m}^{2}$ et 726 $\mathrm{m}^{2}$ ). La majorité des maraichers de Lubumbashi louent les parcelles de culture. En effet, l'urbanisation rapide des villes, due à l'augmentation incessante de leurs 


\section{Useni et al. J. Appl. Biosci. 2014. Problématique de la valorisation agricole des biodéchets dans la ville de Lubumbashi.}

populations, diminue la disponibilité en terres pour des occupations et utilisations du sol telles que l'agriculture (Carole, 2012). Les biodéchets utilisés par les maraichers lushois s'obtiennent, à grande partie, par achat. Ceci traduit le grand intérêt que les maraichers ont découvert en les utilisant. Dans la plupart des cas, les déchets agricoles proviennent de la ferme, car les maraichers prétendent que, les fumiers génèrent relativement plus de fertilité surtout quand il provient fraichement de la ferme. Depuis bien longtemps, le fumier est connu comme l'une des voies efficaces pour améliorer la fertilité et la production des cultures dans les sols tropicaux (Useni et al., 2013). II constitue une source de nutriments $(N, P, K)$ pour les cultures, et son utilisation est la plus courante en zone de maraîchages. Toutefois, la disponibilité du fumier est de plus en plus limitée par rapport au nombre d'exploitants rendant insuffisant tout système d'intensification de l'agriculture (Ruganzu, 2009; Muzingu, 2010). A ce sujet, les résultats montrent que 75,38 , et $80 \%$ des acteurs dans les trois groupe enfuissent les biodéchets lors de l'utilisation, pour simplifier leur dégradation. Cette hypothèse est soutenue par le fait que les fumiers doivent être incorporés immédiatement après l'épandage, faute de quoi, il y aurait perte de la fraction ammoniacale. Ces pertes varient de $30 \%$ dans le cas de fumier et de $70 \%$ dans le cas des autres lisiers (Tejada et al., 2008). Par ailleurs, l'épandage de biodéchets à la surface n'est pas meilleur, il comporte également des risques de propagation de certaines maladies cryptogamiques, de certains rongeurs, et des risques de contamination importante par les graines de mauvaises herbes (Muzingu, 2010). Cependant; d'après les résultats de la présente étude $46,8 \%$; $61,5 \%$ et $80 \%$, de maraichers utilisent les biodéchets sans toute fois les composter ; pratique qui n'est pas à encourager. Ce sont les maraîchers urbains qui s'adonnent à cette forme de valorisation sans compostage. Cette pratique bien que bon marché, entraine par ailleurs de sérieux inconvénients. Elle peut être responsable d'une diminution de la productivité agricole des sols, par l'immobilisation momentanée de l'azote minéral par les microorganismes responsables de la dégradation de la matière organique; dans la mesure où le rapport $\mathrm{C} / \mathrm{N}$ est élevé (Kaboré et al., 2011). Dans cette étude, les groupes I et II ne recourent pas aux biodéchets compostés; car ils ne maîtrisent pas seulement la technique de compostage, mais ils sont également sous-informés. Dans ce contexte, l'utilisation des composts de biodéchets reste très limitée à l'heure actuelle par les différents maraichers rencontrés qui, leurs préfèrent des déchets municipaux. Ceux qui utilisent les composts, font euxmêmes le compostage. Selon Kaboré (2004), rapporte les maraichers du Burkina Faso choisissent de substituer les déchets bruts aux composts, pour des limités de logistique et le manque de temps à allouer à cette activité. En plus de ces constats, ces maraichers ignorent les modes de fabrication du compost (Kaboré, 2004). Les attentes des acteurs sont assez spécifiques pour chaque groupe, elles tournent autour des effets de tout fertilisant organique sur la croissance et le développement des plants, en améliorant les propriétés du sol et en leur fournissant des éléments nutritifs. Certes, la matière organique est d'une importance fondamentale dans la durabilité pour la fertilité des sols ; donc pour une production agricole durable, du fait de ses effets physiques, chimiques et biologiques (FAO, 2005 ; Tejada et al., 2008). Son influence sur les propriétés du sol dépendra de la quantité et du type de matière organique ajoutée (Tejada et al., 2008).

La composition chimique varie en fonction des différents types de biodéchets, cependant le rapport $\mathrm{C} / \mathrm{N}$ appréciable de tous ces types de biodéchets demeure le compost. En se référant à la synthèse de valeurs agronomiques de compost établie par I'ADAESO, la qualité de compost utilisé par ces maraichers est bonne. En effet, le compost de biodéchets est considéré comme un amendement organique dont les teneurs en azote et phosphore sont relativement variables en fonction de la nature des déchets entrant $(0,28 \%$ et $0,133 \%)$. A l'heure actuelle il n'y a pratiquement aucune référence agronomique pour ce dernier. Malgré la grande variabilité du compost de biodechets, celui-ci présente toujours un rapport $\mathrm{C} / \mathrm{N}$ élevé (10 à 11), ce qui fait de lui un bon amendement organique, bien que la seule notion de $\mathrm{C} / \mathrm{N}$ ne suffise pas pour l'affirmer (FAO, 2005). La composition de fumier de poule échantillonné s'écarte de la gamme des valeurs établie par Huot et al. (2003). Ainsi on admet, pour un fumier de poule de bonne qualité, le $\mathrm{C} / \mathrm{N}$ moyen autour de 20 , la teneur en $\mathrm{Ca}$ et $\mathrm{C}$ se situant entre 0,6 et $62 \%$; le taux d'azote de $3,1 \%$. Selon cet auteur, le fumier de poule est considéré comme étant une matière fertilisante à faible capacité amendant, grâce à sa richesse en azote et à la meilleure utilisation de cet azote après épandage. Par ailleurs, la conformité dans ces analyses s'observe au niveau de la teneur en $\mathrm{P}, \mathrm{Mg}$, et en matière organique. Le taux de $\mathrm{P}_{2} \mathrm{O}_{5}$ est moins concentré dans le fumier de 
poule pouvant remonter jusqu'à $0,7 \% ; 36 \%$ de matière organique. Par ailleurs, les fumiers de porcs ont un effet alcalinisant sur le sol, puisque la teneur en $\mathrm{CaO}$ et généralement assez élevée $(2,6 \%)$; le $\mathrm{Mg}$ se retrouve dans une proportion de $7,8 \%$. Alors que ces teneurs en $\mathrm{Ca}$ et $\mathrm{Mg}$ sont le 1/3 de ce qu'a donné le fumier de porc utilisé par les maraichers lushois; de ce fait leur fumier ne convient pas sur sol légèrement acide. Par contre, il y a de faibles teneurs en d'autres éléments analysés par rapport aux valeurs fournies par cet auteur. La teneur en N, P, C, en matière organique, ainsi que le rapport $\mathrm{C} / \mathrm{N}$ sont estimés respectivement

\section{CONCLUSION}

La présente étude s'était proposé d'analyser la problématique de la valorisation des déchets urbains solides, dans un contexte de maraichage à Lubumbashi. Ainsi, une enquête a été réalisée afin de décrire le profil des acteurs, la variabilité des pratiques liée à cette activité ; et vérifier les renseignements par l'analyse des valeurs fertilisantes relatives à chacun des types des biodéchets. Au terme de l'enquête, les maraichers de Lubumbashi sont essentiellement des femmes mariées, âgés de plus de 18 ans, actrices de carrière et ne possédant pas de titre foncier. La moitié des biodéchets utilisés étaient des fumiers de poule et accessoirement le fumier de porc, obtenus par achat et en proviennent de la ferme. La seconde moitié des biodéchets est constitué par les composts, mais encore la paille en faible quantité. Pour l'ensemble de ces déchets, les maraichers ignorent les modes de compostages et préfèrent une application à l'état brut, qu'ils enfouissent dans le sol. Quant à la gestion des résibiodéchets après récolte, le recyclage est le plus pratiqué pour la plupart des maraîchers enquêtés. En

\section{BIBLIOGRAPHIE}

Carole K., 2012. Étude des caractéristiques socioéconomiques et technico-professionnelles des producteurs en lien avec la spatialité de leur activité. Mémoire de DEA inédit. Faculté de Science. Université libre de Bruxelles. 83 p.

De Bon H., Parrot L., Moustier P., 2008. Sustainable urban agriculture in developing countries. A review. Agronomy for Sustainable Development 30:21-32

FAO, 2005. Gestion de la fertilité des sols pour la sécurité alimentaire en Afrique subsaharienne. Rome, Italie, $63 \mathrm{p}$. à : $2,7 \% ; 0,7 \% ; 54 \% ; 31,3 \%$ et $20 \%$. Étant donné les écarts de composition de ce fumier par rapport aux valeurs ci-hauts énumérées, le fumier de porc utilisé par ces maraichers présente moins d'intérêts pour le maraichage. Généralement, la paille est assez pauvre en azote total et dans une moindre mesure en phosphore et potassium. Cependant, la teneur en ces deux éléments peut aller du simple au triple. La paille est riche en calcium et peut posséder des teneurs en magnésium et en oligo-éléments assez intéressantes (Muzingu, 2010).

épandant les biodéchets au sol, les maraichers s'attendent à une augmentation de productivité et une amélioration des propriétés physico-chimiques du sol; alors que la qualité des biodéchets utilisée est à la fois, évaluée à travers l'aspect végétatif des légumes et l'augmentation de rendement. Certes, la composition des types de biodéchets a varié en fonction des éléments considérés, tout comme le rapport $\mathrm{C} / \mathrm{N}$ qui leurs est associé. Ainsi sous réserve de reproduction spatio-temporelle de ces analyses, le compost s'avère le type de biodéchets le plus appréciable, pour autant qu'il définisse le rapport $\mathrm{C} / \mathrm{N}$ dans la gamme recommandée. Eu égard aux résultats de cette étude, nous conseillons vivement aux maraîchers de s'intéresser au compostage comme mode de traitement des biodéchets, pour leur éventuelle utilisation en culture. II convient d'évaluer in situ, les attentes des maraichers quant à ces considérations, de façon à constituer un éventail des renseignements applicables dans la carrière de maraîchage

Frauman, E., 2003. Évolution de l'agriculture périurbaine à Lubumbashi (République Démocratique du Congo) en période de crise. Mémoire présenté en vue de l'obtention du grade de bio-ingénieur à l'Université Libre de Bruxelles.

Houot S., Francou C., Vergé-Leviel C., Michelin J., Bourgeois S., Linères M., Morel $P$., Parnaudeau V., Le Bissonnais Y., Dignac M.F., Dumat C., Cheiab A., Poitrenaud M., 2003. Valeur agronomique et impacts environnements de composts d'origine urbaine 
: variation avec la nature du compost. Dossier de l'environnement de l'INRA 25 :107-124.

Kaboré W-T. T., Hien E., Zombré P., Coulibaly A., Houot S., Masse D., 2011. Valorisation de substrats organiques divers dans l'agriculture péri-urbaine de Ouagadougou (Burkina Faso) pour l'amendement et la fertilisation des sols : acteurs et pratiques. Biotechnol. Agron. Soc. Environ. 15(2) : 271-286

Kaboré.W.T., 2004. Impact de l'apport des déchets urbains solides non triés sur les potentialités agronomiques des sols : cas de l'agriculture périurbaine de Ouagadougou. Mémoire de Fin d'Etude, IDR, Burkina Faso, 78p.

Kalamba, T., Khonde, M., llunga, N. \& Mansinsa, M., 1998. Monographie de la Province $d u$ Katanga. PNUD/UNOPS. Programme national de relance du secteur agricole et rural (PNSAR), p 137.

Kanyankogote, P., Van Ranst, E., Verdoody, A., Baert, G., 2005. Effet de la lave trachybasaltique broyée sur les propriétés chimiques de sols de climat tropical humide. Étude et Gestion des Sols, 12 (4): 301-311.

Kasongo L.E., Mwamba M.T., Tshipoya M.P., Mukalay M.J., Useni S.Y., Mazinga K.M., Nyembo K.L., 2013. Réponse de la culture de soja (Glycine max L. (Merril) à l'apport des biomasses vertes de Tithonia diversifolia (Hemsley) A. Gray comme fumure organique sur un Ferralsol à Lubumbashi, R.D. Congo. Journal of Applied Biosciences 63: 4727 - 4735

Lekane K, 2003. Les relations maraichage-élevage dans la récupération et la valorisation des sous-produits de l'agriculture urbaine et périurbaine : cas de la ville de Yaoundé. Mémoire d'Ingénieur Agronome, Université de Dschang, FASA, UDS, $118 \mathrm{p}$.

Malaisse F., 1990. La couverture végétale de Lubumbashi. In Bruneau J.C., Pain M., (Ed), atlas de Lubumbashi. Edition publidix, université Paris X- Nanterre, pp 30-31

Moustier P., Moumbé M., Huat J., 2004. La gestion concertée et durable des filières maraîchères urbaines. In, Développement durable de l'agriculture urbaine en Afrique francophone. Enjeux, concepts et méthodes. Ed. Olanrewaju B. Smith, Moustier P, Mougeot A.J-L et Fall A, CIRAD/CRDI :81-113.
Mujinya B.B., Mees F., Boeckx P., Bode S., Baert G., Erens H., Delefortrie S., Verdoodt A., Ngongo M.L. \& Van Ranst E., 2011. The origin of carbonate in the termite mounds of the Lubumbashi area, DR Congo. Geoderma, 165: 95-105

Mulaji K.C., 2011. Utilisation des composts de biodéchets ménagers pour l'amélioration de la fertilité des sols acides de la province de Kinshasa (République Démocratique $d u$ Congo). Thèse de doctorat, université de Liège- Gembloux Agro-Biotech, 220p.

Muzingu, B., 2010. Les sites maraichers coopérativisés de Kinshasa en RD Congo. Contraintes environnementales et stratégies des acteurs. Thèse, Faculté des Sciences Economiques, Sociales et Politiques, UCL, Belgique, 169p.

N'Dienor M., 2006. Fertilité et gestion de la fertilisation dans les systèmes maraîchers périurbains des pays en développement : intérêts et limites de la valorisation agricole des déchets urbains dans ces systèmes, cas de l'agglomération d'Antananarivo (Madagascar). Thèse de doctorat, Université d'Antanarivo, École Supérieure des Sciences Agronomiques, 242p + Annexes

Niang S., 1999. Gestion des déchets urbains. L'utilisation des eaux usées brutes dans l'agriculture urbaine au Sénégal : bilan et perspectives. In, Agriculture urbaine en Afrique de l'Ouest. Une contribution à la sécurité alimentaire et à l'assainissement des villes. Ed. Olanrewaju B. Smith, CRDI/CTA, 1999, $240 \mathrm{p}$.

Nkuku C., Rémon M., 2006. Stratégies de survie à Lubumbashi (R-D Congo). Enquête sur 14000 ménages urbains, Archive congolaise, l'Harmattan, Paris, 2006

Ruganzu, V., 2009. Potentiel d'amélioration de la fertilité des sols acides par l'apport de biomasses végétale naturelle fraiche combinées à du travertin au Rwanda. Thèse de doctorat, Gembloux Agro-Bio Tech, Université de Liège, Gembloux, Belgique, $199 \mathrm{p}$.

SENAHUP, 2008. Rapport annuel du service national de l'horticulture urbaine et péri- urbaine à Lubumbashi. RDCongo.

Sys C., Schmitz A., 1959. Notice explicative de la carte des sols et de la végétation. Région 
d'Élisabethville (Haut-Katanga). Bruxelles, Publ. INEAC. 70 p.

Tejada M., Gonzalez J.L., Garcia-Martinez A.M., Parrado J., 2008. Effects of different green manures on soil biological properties and maize yield. Bioressource Technology 99 : 1758-1767.

Useni S.Y., Baboy L.L., Nyembo K.L., Mpundu M.M., 2012. Effets des apports combinés de biodéchets et de fertilisants inorganiques sur le rendement de trois variétés de Zea mays $\mathrm{L}$. cultivées dans la région de Lubumbashi. Journal of Applied Biosciences 54: 39353943
Useni S.Y., Chukiyabo K.M., Tshomba K.J., Muyambo M.E., Kapalanga K.P., Ntumba N.F., Kasangij K.P., Kyungu K., Baboy L.L., Nyembo K.L., Mpundu M.M., 2013. Utilisation des déchets humains recyclés pour l'augmentation de la production du maïs (Zea mays L.) sur un ferralsol du sud-est de la RD Congo. Journal of Applied Biosciences 66:5070 - 5081

Vranken I., 2010. Pollution et contamination des sols aux métaux lourds dues à l'industrie métallurgique à Lubumbashi: Empreinte écologique, impact paysager, pistes de gestion. Travail de fin d'étude. Université Libre de Bruxelles. $118 \mathrm{pp}$. 